\title{
GENERAL ORDER CONDITIONS FOR STOCHASTIC PARTITIONED RUNGE-KUTTA METHODS
}

\author{
SVERRE ANMARKRUD, KRISTIAN DEBRABANT, AND ANNE KVÆRN
}

\begin{abstract}
In this paper stochastic partitioned Runge-Kutta (SPRK) methods are considered. A general order theory for SPRK methods based on stochastic B-series and multicolored, multishaped rooted trees is developed. The theory is applied to prove the order of some known methods, and it is shown how the number of order conditions can be reduced in some special cases, especially that the conditions for preserving quadratic invariants can be used as simplifying assumptions.
\end{abstract}

\section{IntroduCtion}

Whenever the right hand side of an ordinary differential equation (ODE) can be split into different parts with different features, it might be worth trying to solve the different parts by different methods. Distinguished examples are stiff/nonstiff parts solved by implicit/explicit methods, fast/slow parts solved by multirate methods, or partitioned symplectic methods for separable Hamiltonian systems. The latter seems to have been the aim when partitioned methods for stochastic differential equations (SDEs) are constructed, e.g. [23, 6, 18, 14, 19, 13, 29]. Although higher order methods have been constructed, to our knowledge there is still no complete order theory for stochastic partitioned Runge-Kutta (SPRK) methods. Such an order theory based on multicolored, multishaped rooted trees is the main contribution in this paper. The theory is valid for both Itô and Stratonovich SDEs.

Constructing high order methods for SDEs is a notoriously nontrivial task. One problem is the required high order stochastic increments. Another is the huge number of order conditions that have to be fulfilled. For this reason, most methods are constructed for problems with some special structure, like linear SDEs, SDEs with additive noise, or separable SDEs. We will demonstrate how the order theory can be simplified in some of these cases. In particular, Sanz-Serna and Abia [27, 1] have proven that for Runge-Kutta and partitioned Runge-Kutta methods preserving quadratic invariants only order conditions related to rootless trees have to be satisfied. A similar result has been proved for stochastic Runge-Kutta methods in [3. This theory depends on the product rule valid for Stratonovich integrals and is therefore only applicable for Stratonovich SDEs. It will be extended to Stratonovich SPRK methods here.

In this paper we consider a system of stochastic partitioned differential equations with $Q$ partitions and $M$ diffusion terms,

$$
X^{(q)}(t)=x_{0}^{(q)}+\sum_{m=0}^{M} \int_{0}^{t} g_{m}^{(q)}\left(X^{(1)}(s), X^{(2)}(s), \ldots, X^{(Q)}(s)\right) \star \mathrm{d} W_{m}(s), \quad q=1, \ldots, Q,
$$

1991 Mathematics Subject Classification. 65C30, 60H35, 65C20.

Key words and phrases. Stochastic differential equation, partitioned stochastic Runge-Kutta methods, stochastic B-series. 
for which we will also use the abbreviated form

$$
\mathrm{d} X^{(q)}(t)=\sum_{m=0}^{M} g_{m}^{(q)}\left(X^{(1)}(t), X^{(2)}(t), \ldots, X^{(Q)}(t)\right) \star \mathrm{d} W_{m}(t), \quad X^{(q)}(0)=x_{0}^{(q)} .
$$

To simplify the notation the deterministic terms are represented by $m=0$, such that $\mathrm{d} W_{0}(s)=\mathrm{d} s$, while $W_{m}, m=1, \ldots, M$, denote one-dimensional and pairwise independent Wiener processes. The integrals w.r.t. the Wiener processes are interpreted as either Itô integrals, $\star \mathrm{d} W_{m}(s)=\mathrm{d} W_{m}(s)$, or Stratonovich integrals, $\star \mathrm{d} W_{m}(s)=\operatorname{od} W_{m}(s)$. We also define the vector of initial values, $x_{0}=$ $\left[x_{0}^{(1)}, x_{0}^{(2)}, \ldots, x_{0}^{(Q)}\right]$. Furthermore, we assume that the coefficients $g_{m}^{(q)}: \mathbb{R}^{d_{1}} \times \ldots \times \mathbb{R}^{d_{Q}} \rightarrow \mathbb{R}^{d_{q}}$ are sufficiently smooth, and that the conditions of the existence and uniqueness theorem [24] are satisfied. The systems are considered to be autonomous. Nonautonomous systems can be included by extending one of the variables $X^{(q)}, q=1, \ldots, Q$, by $t$, or by considering the equation $t^{\prime}=1$ as a separate partitioning.

Denote the numerical approximation of $X^{(q)}$ at time $t_{n}$ by $Y_{n}^{(q)}$. For the solution of (11) we consider general, $s$-stage SPRK methods given by

$$
\begin{aligned}
H_{i}^{(q)} & =Y_{n}^{(q)}+\sum_{m=0}^{M} \sum_{j=1}^{s} Z_{i, j}^{(q, m)} g_{m}^{(q)}\left(H_{j}^{(1)}, \ldots, H_{j}^{(Q)}\right), \quad i=1, \ldots, s, \\
Y_{n+1}^{(q)} & =Y_{n}^{(q)}+\sum_{m=0}^{M} \sum_{i=1}^{s} \gamma_{i}^{(q, m)} g_{m}^{(q)}\left(H_{i}^{(1)}, \ldots, H_{i}^{(Q)}\right)
\end{aligned}
$$

for partitions $q=1,2, \ldots, Q$. The coefficients $\gamma_{i}^{(q, m)}$ and $Z_{i, j}^{(q, m)}, i, j=1, \ldots, s$, include random variables that depend on the stepsize $h$. The random variables used in each step are assumed to be i.i.d., and also $h$ might change from step to step. To simplify the notation, here and in the following we omit to indicate this by an additional index $n$ on $h$, the random variables used, the coefficients $\gamma^{(q, m)}=\left(\gamma_{i}^{(q, m)}\right)_{i=1}^{s}$ and $Z^{(q, m)}=\left(Z_{i, j}^{(q, m)}\right)_{i, j=1}^{s}$, and the stage values $H_{i}^{(q)}$. This will in particular also hold for the Wiener increments $\Delta W_{m}=\int_{t_{n}}^{t_{n}+h} \mathrm{~d} W_{m}(s)$.

The coefficients of an SPRK method can be gathered in a generalized Butcher tableau. In the frequently encountered case that there exist matrix functions $Z^{(q)}$ and vector functions $\gamma^{(q)}$ such that with some vectors of random variables $\xi_{m}$ it holds that $Z^{(q, m)}=Z^{(q)}\left(\xi_{m}\right)$ and $\gamma^{(q, m)}=\gamma^{(q)}\left(\xi_{m}\right)$ for $m=1, \ldots, M$ and $q=1, \ldots, Q$, we will write the Butcher tableau as follows:

\begin{tabular}{c|c}
$Z^{(1,0)}$ & $Z^{(1, m)}$ \\
\hline$\vdots$ & $\vdots$ \\
\hline$Z^{(Q, 0)}$ & $Z^{(Q, m)} \quad m=1, \ldots, M$. \\
\hline \hline$\left(\gamma^{(1,0)}\right)^{\top}$ & $\left(\gamma^{(1, m)}\right)^{\top}$ \\
\hline$\vdots$ & $\vdots$ \\
\hline$\left(\gamma^{(Q, 0)}\right)^{\top}$ & $\left(\gamma^{(Q, m)}\right)^{\top}$
\end{tabular}

Remark 1. The splitting (11) is sometimes called a horizontal splitting. The results are equally valid for a vertical splitting, that is SDEs split by

$$
\hat{X}(t)=\hat{x}_{0}+\sum_{q=1}^{Q} \sum_{m=0}^{M} \int_{0}^{t} \hat{g}_{m}^{(q)}(\hat{X}(s)) \star \mathrm{d} W_{m}(s), \quad q=1, \ldots, Q,
$$


as (11) can, assuming $d_{1}=\cdots=d_{Q}$ (extending the $X^{(q)}$ by zero components were necessary), be transformed to (4) by

$$
\hat{X}(t)=\sum_{q=1}^{Q} X^{(q)}(t), \quad g_{m}^{(q)}\left(X^{(1)}(t), X^{(2)}(t), \ldots, X^{(Q)}(t)\right)=\hat{g}_{m}^{(q)}\left(\sum_{q=1}^{Q} X^{(q)}(t)\right) .
$$

By summing up the expressions of (3) the corresponding SPRK method becomes

$$
\begin{aligned}
\hat{H}_{i} & =\hat{Y}_{n}+\sum_{q=1}^{Q} \sum_{m=0}^{M} \sum_{j=1}^{s} Z_{i, j}^{(q, m)} \hat{g}_{m}^{(q)}\left(\hat{H}_{j}\right), \quad i=1, \ldots, s, \\
\hat{Y}_{n+1} & =\hat{Y}_{n}+\sum_{q=1}^{Q} \sum_{m=0}^{M} \sum_{i=1}^{s} \gamma_{i}^{(q, m)} \hat{g}_{m}^{(q)}\left(\hat{H}_{i}\right),
\end{aligned}
$$

where $\hat{Y}_{n}$ is the numerical approximation of $\hat{X}\left(t_{n}\right)$.

Example 1. Consider the Langevin equation of motion

$$
\begin{aligned}
& \dot{r}=v, \\
& \dot{v}=f(r, t)-\alpha v+\beta(t),
\end{aligned}
$$

which describes the evolution of a particle with unit mass, coordinate $r(t)$ and velocity $v(t)$. The particle is affected by three forces: $f(r, t)$, a friction force $\alpha v$ for a friction coefficient $\alpha \geq 0$ and thermal white noise $\beta(t)$. Representing the noise term as $\beta W(t)$ with some constant $\beta$, this can be written as a proper SDE, split vertically

$$
\left(\begin{array}{l}
\mathrm{d} R(t) \\
\mathrm{d} V(t)
\end{array}\right)=\underbrace{\left(\begin{array}{c}
V(t) \\
-\alpha V(t)
\end{array}\right)}_{\hat{g}_{0}^{(1)}} \mathrm{d} t+\underbrace{\left(\begin{array}{c}
0 \\
f(R(t), t)
\end{array}\right)}_{\hat{g}_{0}^{(2)}} \mathrm{d} t+\underbrace{\left(\begin{array}{c}
0 \\
\beta
\end{array}\right)}_{\hat{g}_{1}^{(2)}} \mathrm{d} W,
$$

or horizontally by

$$
\begin{aligned}
\left(\begin{array}{c}
\mathrm{d} R(t) \\
\mathrm{d} U_{1}(t)
\end{array}\right) & =\underbrace{\left(\begin{array}{c}
U_{1}(t)+U_{2}(t) \\
-\alpha\left(U_{1}(t)+U_{2}(t)\right)
\end{array}\right)}_{g_{0}^{(1)}} \mathrm{d} t \\
\mathrm{~d} U_{2}(t) & =\underbrace{f(R(t), t)}_{g_{0}^{(2)}} \mathrm{d} t+\underbrace{\beta}_{g_{1}^{(2)}} \mathrm{d} W(t)
\end{aligned}
$$

with $V(t)=U_{1}(t)+U_{2}(t)$.

Grønbech-Jensen and Farago [1] proposed the following scheme to solve the Langevin equation,

$$
\begin{aligned}
& R_{n+1}=R_{n}+b h V_{n}+\frac{b h^{2}}{2} f\left(R_{n}, t_{n}\right)+\frac{b h}{2} \beta \Delta W, \\
& V_{n+1}=a V_{n}+\frac{h}{2}\left(a f\left(R_{n}, t_{n}\right)+f\left(R_{n+1}, t_{n+1}\right)\right)+b \beta \Delta W
\end{aligned}
$$

with

$$
a=\frac{1-\frac{\alpha h}{2}}{1+\frac{\alpha h}{2}}, \quad b=\frac{1}{1+\frac{\alpha h}{2}}
$$


This can be reformulated equivalently as an SPRK method with $\hat{H}_{1}=\left(\begin{array}{c}R_{n} \\ V_{n}\end{array}\right)$ and

$$
\begin{array}{r}
\left(\begin{array}{l}
\hat{H}_{2,1} \\
\hat{H}_{2,2}
\end{array}\right)=\left(\begin{array}{l}
R_{n} \\
V_{n}
\end{array}\right)+\frac{h}{2}\left(\begin{array}{c}
\hat{H}_{1,2}+\hat{H}_{2,2} \\
-\alpha\left(\hat{H}_{1,2}+\hat{H}_{2,2}\right)
\end{array}\right)+h\left(\begin{array}{c}
0 \\
f\left(\hat{H}_{1,1}, t_{n}\right)
\end{array}\right)+\Delta W\left(\begin{array}{l}
0 \\
\beta
\end{array}\right), \\
0 \\
\left(\begin{array}{l}
R_{n+1} \\
V_{n+1}
\end{array}\right)=\left(\begin{array}{l}
R_{n} \\
V_{n}
\end{array}\right)+\frac{h}{2}\left(\begin{array}{c}
\hat{H}_{1,2}+\hat{H}_{2,2} \\
-\alpha\left(\hat{H}_{1,2}+\hat{H}_{2,2}\right)
\end{array}\right)+\frac{h}{2}\left(\begin{array}{c}
0 \\
f\left(\hat{H}_{1,1}, t_{n}\right)+f\left(\hat{H}_{2,1}, t_{n+1}\right)
\end{array}\right) \\
+\Delta W\left(\begin{array}{l}
0 \\
\beta
\end{array}\right),
\end{array}
$$

where $\hat{H}_{i, j}$ denotes component $j$ of $\hat{H}_{i}$. Due to the special structure of the problem, the scheme (8) can be interpreted as an application of several SPRK methods (with $M=1$ ), of which we mention the following two:

\begin{tabular}{cc|cc}
0 & 0 & 0 & 0 \\
$\frac{h}{2}$ & $\frac{h}{2}$ & 0 & 0 \\
\hline 0 & 0 & 0 & 0 \\
$h$ & 0 & $\Delta W_{m}$ & 0 \\
\hline \hline$\frac{h}{2}$ & $\frac{h}{2}$ & 0 & 0 \\
\hline$\frac{h}{2}$ & $\frac{h}{2}$ & $\Delta W_{m}$ & 0
\end{tabular},

\begin{tabular}{cc|cc}
0 & 0 & 0 & 0 \\
$\frac{h}{2}$ & $\frac{h}{2}$ & $\frac{\Delta W_{m}}{2}$ & $\frac{\Delta W_{m}}{2}$ \\
\hline 0 & 0 & 0 & 0 \\
$h$ & 0 & $\Delta W_{m}$ & 0 \\
\hline \hline$\frac{h}{2}$ & $\frac{h}{2}$ & $\frac{\Delta W_{m}}{2}$ & $\frac{\Delta W_{m}}{2}$ \\
\hline$\frac{h}{2}$ & $\frac{h}{2}$ & $\frac{\Delta W_{m}}{2}$ & $\frac{\Delta W_{m}}{2}$
\end{tabular}$\quad m=1, \ldots, M$.

While the method to the left might be the obvious choice, it is only convergent for partitioned problems for which there is no noise in the first partitioning. In this particular case it coincides with the method to the right. In Section 3.1 we will prove this method to be of strong order 1 and weak order 2 when applied to more general partitioned SDEs with additive noise.

In Section 2, a general order theory for SPRKs is developed. The theory is based on stochastic B-series and multicolored, multishaped rooted trees. In Section 3 two particular cases are studied: SDEs with additive noise and separable problems. We will here present examples of how the Bseries theory can be used to find the order of some given methods. In Section 4 it is shown how the number of order conditions can be further reduced in the case of methods preserving quadratic invariants of Stratonovich SDEs.

\section{ORDER THEORY}

B-series for deterministic ODEs were introduced by J. C. Butcher 8 in 1963. B-series for SDEs were developed by Burrage and Burrage [4, 5, 7] for strong convergence of Stratonovich SDEs, by Komori, Mitsui and Sugiura [17] and Komori [16] for weak convergence of Stratonovich SDEs, and by Rößler [25, 26] for weak convergence in both the Itô and Stratonovich case. A unified theory for B-series encompassing both weak and strong convergence for both Itô and Stratonovich SDEs was given in [9. In the following we will generalize this to SPRKs.

Our first goal is to find B-series representations of (3), and we begin by assuming $X^{(q)}(h)$ can be written as a B-series $B^{(q)}\left(\phi, x_{0} ; h\right)$,

$$
B^{(q)}\left(\phi, x_{0} ; h\right)=\sum_{\tau \in T_{q}} \alpha(\tau) \cdot \phi(\tau)(h) \cdot F(\tau)\left(x_{0}\right),
$$

where $T_{q}$ is the set of shaped, colored, rooted trees as defined below. The terms $\alpha(\tau)$ are combinatoric terms. The elementary weight functions $\phi(\tau)(h)$ are stochastic integrals or random variables, and $F(\tau)\left(x_{0}\right)$ are the elementary differentials. To simplify the presentation, we assume that all 


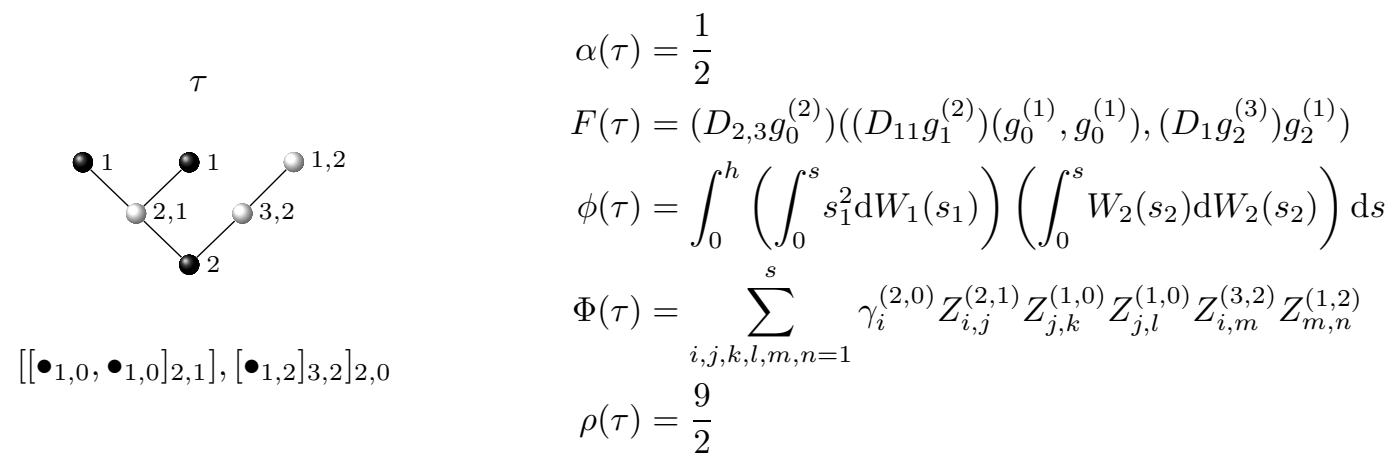

Figure 1. An example of a shaped, colored, rooted tree and its corresponding functions.

elementary differentials exist and all considered B-series converge. Otherwise, one has to consider truncated B-series and discuss the remainder term [25].

Definition 1 (Trees and combinatorial coefficients). The set of shaped, rooted trees

$$
T=T_{1} \cup T_{2} \cup \cdots \cup T_{Q}
$$

where

$$
T_{q}=\left\{\emptyset_{q}\right\} \cup T_{q, 0} \cup T_{q, 1} \cup \cdots \cup T_{q, M}
$$

for $q=1, \ldots, Q$ is recursively defined as follows:

(i) The graph $\bullet_{q, m}$ with only one vertex of shape $q$ and color $m$ belongs to $T_{q, m}$.

(ii) If $\tau_{1}, \tau_{2}, \ldots, \tau_{\kappa} \in T \backslash\left\{\emptyset_{1}, \ldots, \emptyset_{Q}\right\}$, then $\left[\tau_{1}, \tau_{2}, \ldots, \tau_{\kappa}\right]_{q, m} \in T_{q, m}$, where $\left[\tau_{1}, \tau_{2}, \ldots, \tau_{\kappa}\right]_{q, m}$ denotes the tree formed by joining the subtrees $\tau_{1}, \tau_{2}, \ldots, \tau_{\kappa}$ each by a single branch to a common root of shape $q$ and color $m$.

Further, we define $\alpha(\tau)$ as

$$
\alpha\left(\emptyset_{q}\right)=1, \alpha\left(\bullet_{q, m}\right)=1, \alpha\left(\tau=\left[\tau_{1}, \ldots, \tau_{\kappa}\right]_{q, m}\right)=\frac{1}{r_{1} ! r_{2} ! \ldots r_{R} !} \prod_{k=1}^{\kappa} \alpha\left(\tau_{k}\right),
$$

where $r_{1}, r_{2}, \ldots, r_{R}$ count equal trees among $\tau_{1}, \tau_{2}, \ldots, \tau_{\kappa}$.

Remark 2. Rooted trees can be represented in the bracket notation, as used in the definition, or illustrated as graphs (see Fig. 11). To ease the reading, deterministic nodes are in the latter represented as black nodes, with the $m=0$ omitted, while stochastic nodes are white. Figure 1 also gives the corresponding examples for the functions of trees that will be defined in the following.

Definition 2 (Elementary differentials). For a tree $\tau \in T$ the elementary differential is a mapping $F(\tau): \mathbb{R}^{d_{1}} \times \ldots \times \mathbb{R}^{d_{Q}} \rightarrow \mathbb{R}^{d}$ defined recursively by

(i) $F\left(\emptyset_{q}\right)\left(x_{0}\right)=x_{0}^{(q)}, \emptyset_{q} \in T_{q}$,

(ii) $F\left(\bullet_{q, m}\right)\left(x_{0}\right)=g_{m}^{(q)}\left(x_{0}\right)$,

(iii) If $\tau=\left[\tau_{1}, \tau_{2}, \ldots, \tau_{\kappa}\right]_{q, m} \in T_{q, m}$, then

$$
F(\tau)\left(x_{0}\right)=\left(D_{q_{1} \ldots q_{\kappa}} g_{m}^{(q)}\right)\left(x_{0}\right)\left(F\left(\tau_{1}\right)\left(x_{0}\right), F\left(\tau_{2}\right)\left(x_{0}\right), \ldots, F\left(\tau_{\kappa}\right)\left(x_{0}\right)\right)
$$

where $q_{k}$ is the shape of $\tau_{k}, k=1, \ldots, \kappa$, and $D_{q_{1} \ldots q_{\kappa}}=\frac{\partial^{\kappa}}{\partial x^{q_{1} \ldots \partial x^{q_{\kappa}}}}$ denotes the derivative operator of order $\kappa$. 
Fundamental for this work is the following lemma which says that if $Y^{(q)}(h)$ can be written as a B-series, then $f\left(Y^{(1)}(h), \ldots, Y^{(Q)}(h)\right)$ can also be written as a B-series. This is a trivial extension of the lemma found in [9].

Lemma 1. If $Y^{(q)}(h)=B^{(q)}\left(\phi, x_{0} ; h\right), q=1, \ldots, Q$, are some B-series and $f \in C^{\infty}\left(\mathbb{R}^{d_{1}} \times \ldots \times\right.$ $\left.\mathbb{R}^{d_{Q}}, \mathbb{R}^{d}\right)$, then $f\left(Y^{(1)}(h), \ldots, Y^{(Q)}(h)\right)$ can be written as a formal series of the form

$$
f\left(Y^{(1)}(h), \ldots, Y^{(Q)}(h)\right)=\sum_{u \in U_{f}} \beta(u) \cdot \psi_{\phi}(u)(h) \cdot G(u)\left(x_{0}\right),
$$

where

(i) $U_{f}$ is a set of trees derived from $T$, by $\bullet_{f} \in U_{f}$, and if $\tau_{1}, \tau_{2}, \ldots, \tau_{\kappa} \in T \backslash\left\{\emptyset_{1}, \ldots, \emptyset_{Q}\right\}$, then $\left[\tau_{1}, \tau_{2}, \ldots, \tau_{\kappa}\right]_{f} \in U_{f}$.

(ii) $G\left(\bullet_{f}\right)\left(x_{0}\right)=f\left(x_{0}\right)$ and

$$
G\left(\left[\tau_{1}, \tau_{2}, \ldots, \tau_{\kappa}\right]_{f}\right)\left(x_{0}\right)=\left(D_{q_{1}, \ldots, q_{\kappa}} f\right)\left(x_{0}\right)\left(F\left(\tau_{1}\right)\left(x_{0}\right), \ldots, F\left(\tau_{\kappa}\right)\left(x_{0}\right)\right) .
$$

(iii) $\beta(\bullet f)=1$ and

$$
\beta\left(\left[\tau_{1}, \tau_{2}, \ldots, \tau_{\kappa}\right]_{f}\right)=\frac{1}{r_{1} ! r_{2} ! \cdots r_{q} !} \prod_{k=1}^{\kappa} \alpha\left(\tau_{k}\right),
$$

where $r_{1}, r_{2}, \ldots, r_{q}$ count equal trees among $\tau_{1}, \tau_{2}, \ldots, \tau_{\kappa}$.

(iv) $\psi_{\phi}(\bullet f)(h)=1$ and $\psi_{\phi}\left(\left[\tau_{1}, \tau_{2}, \ldots, \tau_{\kappa}\right]_{f}\right)(h)=\prod_{k=1}^{\kappa} \phi\left(\tau_{k}\right)(h)$.

If we apply Lemma 1 to the functions $g_{m}^{(q)}$ in (1) we get

$$
g_{m}^{(q)}\left(X^{(1)}(h), \ldots, X^{(Q)}(h)\right)=\sum_{u \in U_{g_{m}^{(q)}}} \beta(u) \cdot \psi_{\phi}(u)(h) \cdot G(u)\left(x_{0}\right) .
$$

By the definitions of trees, $T_{q, m}$, and elementary differentials, $F(\tau)\left(x_{0}\right)$, we can write this as

$$
g_{m}^{(q)}\left(X^{(1)}(h), \ldots, X^{(Q)}(h)\right)=\sum_{\tau \in T_{q, m}} \alpha(\tau) \cdot \phi_{q, m}^{\prime}(\tau)(h) \cdot F(\tau)\left(x_{0}\right),
$$

where

$$
\phi_{q, m}^{\prime}(\tau)(h)= \begin{cases}1, & \text { if } \tau=\bullet_{q, m} \\ \prod_{k=1}^{\kappa} \phi\left(\tau_{k}\right)(h), & \text { if } \tau=\left[\tau_{1}, \ldots, \tau_{\kappa}\right]_{q, m} \in T_{q, m}\end{cases}
$$

We now write the exact solutions of (1) as B-series and use (11) to obtain

$$
\begin{aligned}
& \sum_{\tau \in T_{q}} \alpha(\tau) \cdot \phi(\tau)(h) \cdot F(\tau)\left(x_{0}\right)= \\
& \\
& x_{0}^{(q)}+\sum_{m=0}^{M} \sum_{\tau \in T_{q, m}} \alpha(\tau) \cdot \int_{0}^{h} \phi_{q, m}^{\prime}(\tau)(s) \star \mathrm{d} W_{m}(s) F(\tau)\left(x_{0}\right) .
\end{aligned}
$$

Comparing term by term we see that

$$
\begin{array}{r}
\phi\left(\emptyset_{q}\right)(h)=1, \text { and } \phi(\tau)(h)=\int_{0}^{h} \phi_{q, m}^{\prime}(\tau)(s) \star \mathrm{d} W_{m}(s) \\
\quad \text { for all } \tau \in T_{q, m}, m=0,1, \ldots, M, q=1, \ldots, Q .
\end{array}
$$

With induction on the height of $\tau$ we have proven the following theorem. 
Theorem 1. The exact solutions $X^{(q)}(h)$ of (1) $, q=1, \ldots, Q$, can be written as $B$-series $B^{(q)}\left(\phi, x_{0} ; h\right)$ with

$$
\begin{aligned}
& \qquad\left(\emptyset_{q}\right)(h)=1, \quad \phi\left(\bullet_{q, m}\right)(h)=\Delta W_{m}(h), \\
& \phi\left(\left[\tau_{1}, \tau_{2}, \ldots, \tau_{\kappa}\right]_{q, m}\right)(h)=\int_{0}^{h} \prod_{j=1}^{\kappa} \phi\left(\tau_{j}(s)\right) \star \mathrm{d} W_{m}(s), \\
& \quad \text { for all }\left[\tau_{1}, \tau_{2}, \ldots, \tau_{\kappa}\right]_{q, m} \in T_{q, m}, q=1, \ldots, Q, m=0,1, \ldots, M .
\end{aligned}
$$

A similar result can be found for the numerical solution of (1) by the $s$-stage SPRK method (3).

Theorem 2. The numerical solutions $Y_{1}^{(q)}$ as well as the stage values can be written in terms of $B$-series

$$
H_{i}^{(q)}=B^{(q)}\left(\Psi_{i}, x_{0} ; h\right), \quad Y_{1}^{(q)}=B^{(q)}\left(\Phi, x_{0} ; h\right)
$$

for all $i=1, \ldots, s, q=1, \ldots, Q$, with

$$
\begin{aligned}
& \Psi_{i}\left(\emptyset_{q}\right)(h)=1, \quad \Psi_{i}\left(\bullet_{q, m}\right)(h)=\sum_{j=1}^{s} Z_{i, j}^{(q, m)}, \\
& \Psi_{i}\left(\left[\tau_{1}, \ldots, \tau_{\kappa}\right]_{q, m}\right)(h)=\sum_{j=1}^{s} Z_{i, j}^{(q, m)} \prod_{k=1}^{\kappa} \Psi_{j}\left(\tau_{k}\right)(h)
\end{aligned}
$$

and

$$
\begin{gathered}
\Phi\left(\emptyset_{q}\right)(h)=1, \quad \Phi\left(\bullet_{q, m}\right)(h)=\sum_{i=1}^{s} \gamma_{i}^{(q, m)}, \\
\Phi\left(\tau=\left[\tau_{1}, \ldots, \tau_{\kappa}\right]_{q, m}\right)(h)=\sum_{i=1}^{s} \gamma_{i}^{(q, m)} \prod_{k=1}^{\kappa} \Psi_{i}\left(\tau_{k}\right)(h) .
\end{gathered}
$$

Proof. Write $H_{i}^{(q)}$ as B-series,

$$
H_{i}^{(q)}=\sum_{\tau \in T_{q}} \alpha(\tau) \Psi_{i}(\tau)(h) F(\tau)\left(x_{0}\right),
$$

for $i=1, \ldots, s, q=1, \ldots, Q$, where as usual the product of vectors is understood componentwise. Inserted into (3a) and using (11) this gives

$$
H_{i}^{(q)}=x_{0}^{(q)}+\sum_{m=0}^{M} \sum_{j=1}^{s} \sum_{\tau \in T_{q, m}} \alpha(\tau)\left(Z_{i, j}^{(q, m)} \cdot \Psi_{j, q, m}^{\prime}(\tau)(h)\right) F(\tau)\left(x_{0}\right) .
$$

A term by term comparison yields (12). The proof of (13) is similar.

The local order of accuracy of the SPRK method can now be decided by comparing the B-series of the exact and the numerical solution. Applying a time transformation, it is hereby sufficient to consider B-series expansions around $t=0$, as done in Theorems 1 and 2, First, we need to define the tree order.

Definition 3. The order of a tree $\tau \in T$ is defined by

$$
\rho\left(\emptyset_{q}\right)=0, \quad \rho\left(\tau=\left[\tau_{1}, \ldots, \tau_{\kappa}\right]_{q, m}\right)=\sum_{k=1}^{\kappa} \rho\left(\tau_{k}\right)+\left\{\begin{array}{l}
1 \text { for } m=0 \\
\frac{1}{2} \text { otherwise }
\end{array}\right.
$$


The following theorem relates the global order of accuracy to the local order. Here, we assume that method (3) is constructed such that $\Phi(\tau)(h)=\mathcal{O}\left(h^{\rho(\tau)}\right)$ for all $\tau \in T$ for mean square convergence, respectively $E \prod_{k=1}^{\kappa} \Phi\left(\tau_{k}\right)=\mathcal{O}\left(h^{\sum_{k=1}^{\kappa} \rho\left(\tau_{k}\right)}\right)$ for all $\tau_{1}, \ldots, \tau_{\kappa} \in T, \kappa \in \mathbb{N}$, for weak convergence.

Theorem 3. The method has mean square global order $p$ if

$$
\begin{array}{cl}
\Phi(\tau)(h)=\phi(\tau)(h)+\mathcal{O}\left(h^{p+\frac{1}{2}}\right), & \forall \tau \in T \text { with } \rho(\tau) \leq p, \\
E \Phi(\tau)(h)=E \phi(\tau)(h)+\mathcal{O}\left(h^{p+1}\right), & \forall \tau \in T \text { with } \rho(\tau) \leq p+\frac{1}{2},
\end{array}
$$

and weak consistency of order $p$ if and only if

$$
E \prod_{k=1}^{\kappa} \Phi\left(\tau_{k}\right)=E \prod_{k=1}^{\kappa} \phi\left(\tau_{k}\right)+\mathcal{O}\left(h^{p+1}\right) \quad \text { whenever } \quad \sum_{k=1}^{\kappa} \rho\left(\tau_{k}\right) \leq p+\frac{1}{2} .
$$

Here, the $\mathcal{O}(\cdot)$-notation refers to $h \rightarrow 0$ and, especially in (14a), to the $L^{2}$-norm. The result (14) was first proved in [5], while (15) is a consequence of a result of Milstein [22, see [9] for details. A list of all trees with $\rho(\tau) \leq 2$ and their corresponding functions are given in Appendix $\mathrm{A}$.

\section{Two SPECIAL CASES}

The amount of order conditions to be satisfied for higher order methods is quite overwhelming. E. g. for a method of strong order 1.5 with $Q=2$ and scalar noise, order conditions for 122 different trees need to be satisfied (some of them being trivially fulfilled, though).

The virtue of partitioned methods becomes clear when applied to problems for which some underlying structure can be exploited, in the sense that many elementary differentials will be zero, and the corresponding trees can thus be ignored in the B-series. The main task is to identify those trees. In this subsection, the idea will be demonstrated with two distinguished examples from Milstein et. al [20, 23, 21], SDEs with additive noise and separable systems, both with two partitionings.

3.1. SDEs with additive noise. We consider partitioned problems with $Q=2$ with additive noise, thus

$$
\begin{aligned}
& \mathrm{d} X^{(1)}=g_{0}^{(1)}\left(X^{(1)}, X^{(2)}, t\right) \mathrm{d} t+\sum_{m=1}^{M} g_{m}^{(1)}(t) \mathrm{d} W_{m}(t), \\
& \mathrm{d} X^{(2)}=g_{0}^{(2)}\left(X^{(1)}, X^{(2)}, t\right) \mathrm{d} t+\sum_{m=1}^{M} g_{m}^{(2)}(t) \mathrm{d} W_{m}(t) .
\end{aligned}
$$

To deal with the nonautonomous case, let us include a third partition:

$$
\mathrm{d} X^{(3)}=1 \mathrm{~d} t
$$

The problem structure induces that many elementary differentials will be zero, and the corresponding trees can thus be ignored in the B-series. Restricting to nonvanishing elementary differentials, we only need to consider trees characterized by the following properties:

- There are no nodes $\bullet_{3, m}$ for $m \neq 0$, since the corresponding $g_{m}^{(3)}=0$.

- Nodes $\bullet_{3,0}$ have no branches, since $g_{0}^{(3)}$ is constant.

- The stochastic nodes $\bullet_{q, m}$ with $q=1,2$ and $m \neq 0$ can only be followed by one or more nodes $\bullet 3,0$, since $g_{m}^{(q)}$ is only time-dependent. 
TABLE 1. Relevant trees up to order two and corresponding functions for twopartitioned SDEs with additive noise (16). Here, $q_{1}, q_{2}, q_{3} \in\{1,2\}$, while $\tilde{q}_{1} \in$ $\{1,2,3\}$. The weights $\Phi(\tau)$ correspond to method (9) extended by $Z^{(3,0)}=Z^{(2,0)}$ and $\gamma^{(3,0)}=\gamma^{(2,0)}$.

\begin{tabular}{|c|c|c|c|c|}
\hline No & $\tau$ & $\rho(\tau)$ & $\phi(\tau)$ & $\Phi(\tau)$ \\
\hline 1 & $q_{1}, m_{1}$ & $\frac{1}{2}$ & $\Delta W_{m_{1}}$ & $\Delta W_{m_{1}}$ \\
\hline 2 & - $\tilde{q}_{1}$ & 1 & $h$ & $h$ \\
\hline 6 & $\int_{q_{1}, m_{1}}^{3}$ & $\frac{3}{2}$ & $\int_{0}^{h} s \mathrm{~d} W_{m_{1}}(s)$ & $\frac{1}{2} h \Delta W_{m_{1}}$ \\
\hline 7 & $\underbrace{q_{2}, m_{2}}_{q_{1}}$ & $\frac{3}{2}$ & $\int_{0}^{h} W_{m_{2}}(s) \mathrm{d} s$ & $\frac{1}{2} h \Delta W_{m_{2}}$ \\
\hline 8 & $q_{1}$ & 2 & $\frac{1}{2} h^{2}$ & $\frac{1}{2} h^{2}$ \\
\hline 9 & $\underbrace{q_{2}, m_{2}}_{q_{1}} \rho^{q_{3}, m_{3}}$ & 2 & $\int_{0}^{h} W_{m_{2}}(s) W_{m_{3}}(s) \mathrm{d} s$ & $\frac{1}{2} h \Delta W_{m_{2}} \Delta W_{m_{3}}$ \\
\hline
\end{tabular}

Of the trees listed in the appendix, this leaves us with the trees no. 1, 2, 6, 7, 8 and 9 and even those can be simplified since $q_{1} \neq 3$ for the trees no. $6,7,8$ and 9 , and $q_{2}=3$ for tree no. 6 . The trees together with their corresponding functions are listed in Table 1 .

Let the SDE (16) be solved by method (9). For the third partition, we use $Z^{(3,0)}=Z^{(2,0)}$ and $\gamma^{(3,0)}=\gamma^{(2,0)}$. The corresponding weights $\Phi(\tau)$ are given in Table 1 . From Theorem 3 we can conclude that the method is of strong order 1, as the order conditions for the trees no. 6 and 7 are only fulfilled in expectation, and that it is of weak order 2 .

The Langevin equation (6) of Example 1 is an example of an SDE (16), thus we can conclude that the method proposed in [11] is of strong order 1 and weak order 2. A further example of an SDE (16) is the following:

Example 2 (Stochastic version of the Jansen and Rit Neural Mass Model). Let $X_{0}, X_{1}$ and $X_{2}$ describe the mean postsynaptic potentials of the main neural population, the excitatory and inhibitory interneurons, respectively. The following model has been proposed in [2]:

$$
\begin{aligned}
\mathrm{d} X_{i}(t) & =X_{i+3}(t), \quad i \in\{0,1,2\} \\
\mathrm{d} X_{3}(t) & =\left[A a\left[\mu_{3}(t)+\operatorname{Sigm}\left(X_{1}(t)-X_{2}(t)\right)\right]-2 a X_{3}(t)-a^{2} X_{0}(t)\right]+\sigma_{3}(t) \mathrm{d} W_{3}(t), \\
\mathrm{d} X_{4}(t) & =\left[A a\left[\mu_{4}(t)+C_{2} \operatorname{Sigm}\left(C_{1} X_{0}(t)\right)\right]-2 a X_{4}(t)-a^{2} X_{1}(t)\right]+\sigma_{4}(t) \mathrm{d} W_{4}(t), \\
\mathrm{d} X_{5}(t) & =\left[B b\left[\mu_{5}(t)+C_{4} \operatorname{Sigm}\left(C_{3} X_{0}(t)\right)\right]-2 b X_{5}(t)-b^{2} X_{2}(t)\right]+\sigma_{5}(t) \mathrm{d} W_{5}(t),
\end{aligned}
$$

where $\operatorname{Sigm}(x)=\frac{\nu_{\max }}{1+e^{r\left(\nu_{0}-x\right)}}, \mu_{i}$ and $\sigma_{i}, i \in\{3,4,5\}$, describe external input and the scaling of the stochastic components, respectively, and $A, B, a, b, C_{i}, i \in\{1,2,3,4\}, r$ and $\nu_{0}, \nu_{\max }$ are parameters. 
3.2. Separable systems. Consider the following system, which typically can arise from separable Hamiltonian systems, discussed e.g. in [21, 4.2.2]:

$$
\begin{aligned}
& \mathrm{d} X^{(1)}(t)=g_{0}^{(1)}\left(X^{(2)}(t)\right) \mathrm{d} t+\sum_{m=1}^{M} g_{m}^{(1)}\left(X^{(2)}(t)\right) \star \mathrm{d} W_{m}(t), \\
& \mathrm{d} X^{(2)}(t)=g_{0}^{(2)}\left(X^{(1)}(t)\right) \mathrm{d} t .
\end{aligned}
$$

The elementary differentials corresponding to the following trees vanish:

- All trees for which a node of shape $q(q \in\{1,2\})$ is followed by a node of the same shape, as $\frac{\partial g_{m}^{(q)}}{\partial x^{(q)}}=0$ for $m=0, \ldots, M$.

- All trees with nodes $\bullet_{2, m}$ with $m \neq 0$ : There is no noise in the second partition, so $g_{m}^{(2)}=0$ for $m=1, \ldots, M$.

The remaining trees $\tau$ with $\rho(\tau) \leq 2$ are then (for $q_{1}, q_{2} \in\{1,2\}$ with $q_{1} \neq q_{2}$ )

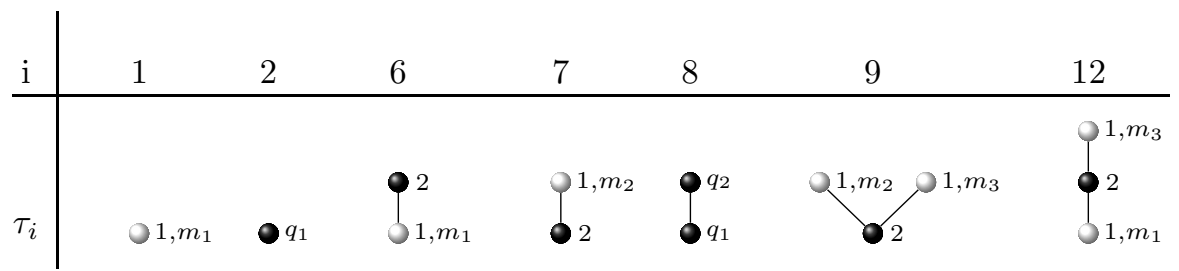

Consider the following method, proposed in [23]:

\begin{tabular}{cc|cc}
$\frac{h}{4}$ & 0 & $\frac{3 J_{(m, 0)}}{2 h}-\frac{\Delta W_{m}}{2}$ & 0 \\
$\frac{h}{4}$ & $\frac{3 h}{4}$ & $\frac{3 J_{(m, 0)}}{2 h}-\frac{\Delta W_{m}}{2}$ & $-\frac{3 J_{(m, 0)}}{2 h}+\frac{3 \Delta W_{m}}{2}$ \\
\hline 0 & 0 & & \\
$\frac{2 h}{3}$ & 0 & & \\
\hline \hline$\frac{h}{4}$ & $\frac{3 h}{4}$ & $\frac{3 J_{(m, 0)}}{2 h}-\frac{\Delta W_{m}}{2}$ & $-\frac{3 J_{(m, 0)}}{2 h}+\frac{3 \Delta W_{m}}{2}$ \\
\hline$\frac{2 h}{3}$ & $\frac{h}{3}$ & &
\end{tabular}

where $J_{(m, 0)}=\int_{t_{n}}^{t_{n}+h}\left(W_{m}(s)-W_{m}\left(t_{n}\right)\right) \mathrm{d} s$. It is straightforward to show that $\phi(\tau)=\Phi(\tau)$ for trees $\tau_{1}, \tau_{2}, \tau_{7}, \tau_{8}$ in (18). Using the fact (see e.g. [15, 10]) that $J_{(m, 0)}+J_{(0, m)}=h \Delta W_{m}$, where $J_{(0, m)}=\int_{t_{n}}^{t_{n}+h}\left(s-t_{n}\right) \star \mathrm{d} W_{m}(s)$, we obtain for $\tau_{6}$ that (remember that for the consistency analysis it is enough to consider the first step of the method, so $t_{n}=t_{0}=0$ )

$$
\begin{aligned}
\Phi\left(\tau_{6}\right) & =\sum_{i=1}^{2} \gamma_{i}^{\left(1, m_{1}\right)} \sum_{j=1}^{2} Z_{i, j}^{(2,0)}=\gamma_{2}^{\left(1, m_{1}\right)} \sum_{j=1}^{2} Z_{2, j}^{(2,0)} \\
& =\left(-\frac{3 J_{\left(m_{1}, 0\right)}}{2 h}+\frac{3 \Delta W_{m_{1}}}{2}\right) \frac{2 h}{3}=-J_{\left(m_{1}, 0\right)}+\Delta W_{m_{1}} h \\
& =J_{\left(0, m_{1}\right)}=\phi\left(\tau_{6}\right) .
\end{aligned}
$$


For the remaining two trees we get:

$$
\begin{aligned}
\Phi\left(\tau_{9}\right) & =\frac{1}{2}\left(\frac{3}{h} J_{\left(m_{2}, 0\right)} J_{\left(m_{3}, 0\right)}-\Delta W_{m_{2}} J_{\left(m_{3}, 0\right)}-\Delta W_{m_{3}} J_{\left(m_{2}, 0\right)}+h \Delta W_{m_{2}} \Delta W_{m_{3}}\right), \\
\Phi\left(\tau_{12}\right) & =\frac{1}{2}\left(-\frac{3}{h} J_{\left(m_{1}, 0\right)} J_{\left(m_{3}, 0\right)}+3 \Delta W_{m_{1}} J_{\left(m_{3}, 0\right)}+\Delta W_{m_{3}} J_{\left(m_{1}, 0\right)}-h \Delta W_{m_{1}} \Delta W_{m_{3}}\right) .
\end{aligned}
$$

From Theorem 3 we can conclude that the method is (remarkably both for Stratonovich and Itô SDEs) of strong order 1.5, since

$$
E \Phi\left(\tau_{9}\right)=E \phi\left(\tau_{9}\right)=\left\{\begin{array}{ll}
\frac{h^{2}}{2} & \text { if } m_{2}=m_{3} \\
0 & \text { otherwise }
\end{array}, \quad E \Phi\left(\tau_{12}\right)=E \phi\left(\tau_{12}\right)=0,\right.
$$

which follows from the following relations:

$$
E \Delta W_{m}^{2}=h, \quad E \Delta W_{m} J_{(m, 0)}=\frac{h^{2}}{2}, \quad E J_{(m, 0)}^{2}=\frac{h^{3}}{3} .
$$

This is of course in accordance with the order result given (for the Stratonovich case) in 20, Theorem 4.3].

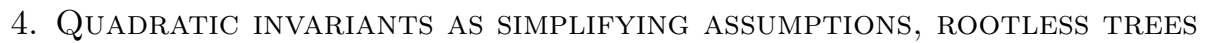

In the previous section, we demonstrated how the special structure of the SDEs can be exploited, in the sense that the elementary differentials corresponding to certain trees are zero, and the corresponding conditions for these trees do not need to be fulfilled. In this section, we will see how for separable Stratonovich SDEs the algebraic relation between the method coefficients that implies the method to preserve quadratic invariants creates certain equivalence classes of trees in the sense that only one condition for each class has to be fulfilled. This is a generalization of a result obtained for deterministic partitioned ODEs by Abia and Sanz-Serna [1. It is similar to the nonpartitioned case, which for ODEs is discussed by Sanz-Serna and Abia 27] and generalized to SDEs in Anmarkrud and Kværnø [3. The conditions for preserving quadratic invariants for non-separable equations with two partitionings have been developed by Hong, $\mathrm{Xu}$ and Wang [19], see also Ma and Ding [19], both in the context of symplectic methods. Using similar ideas, it is possible to find conditions for a more general partitioning. However, the extra freedom gained by more partitionings is to some extent lost by the huge number of extra conditions that have to be satisfied. In this paper, the discussion is restricted to separable equations with the number of partitions of (1) to be two, i.e.

$$
\begin{aligned}
& \mathrm{d} X^{(1)}(t)=\sum_{m=0}^{M} g_{m}^{(1)}\left(X^{(2)}(t)\right) \circ \mathrm{d} W_{m}(t), \\
& \mathrm{d} X^{(2)}(t)=\sum_{m=0}^{M} g_{m}^{(2)}\left(X^{(1)}(t)\right) \circ \mathrm{d} W_{m}(t),
\end{aligned}
$$

which is also the stochastic counterpart to the system discussed in [1].

We assume that the system has a quadratic invariant $I\left(X^{(1)}, X^{(2)}\right)=X^{(1)^{\top}} D X^{(2)}$ for a matrix $D$ of the appropriate dimension and arbitrary initial values $X^{(1)}(0), X^{(2)}(0)$.

Example 3 (Synchrotron oscillations). Consider a stochastically perturbed Hamiltonian system of a pendulum (28], where we set $\lambda_{A}=0$ ) with Hamiltonians

$$
H_{0}(p, x, t)=\frac{p^{2}}{2}, \quad H_{1}(p, x, t)=\omega^{2} \sin (x) \lambda_{P h} .
$$




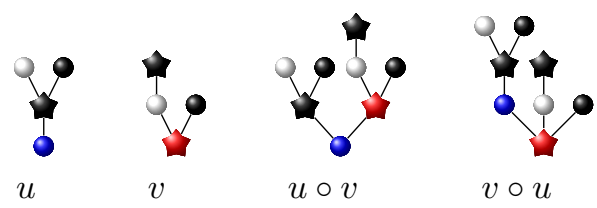

Figure 2. Demonstration of the Butcher product (with real colors and shapes instead of indices).

The resulting SDE system

$$
d p=-\omega^{2} \sin (x) d t-\lambda_{P h} \omega^{2} \cos (x) \circ d W(t), \quad d x=p d t
$$

preserves symplecticity, i.e. a quadratic invariant [21, Section 4.1].

As in [12, chapter IV.2.2] one can prove the following theorem:

Theorem 4. If the coefficients of the SPRK method (3) satisfy

$$
\begin{aligned}
\gamma_{i}^{\left(1, m_{1}\right)} \gamma_{j}^{\left(2, m_{2}\right)}=\gamma_{j}^{\left(2, m_{2}\right)} Z_{j, i}^{\left(1, m_{1}\right)}+\gamma_{i}^{\left(1, m_{1}\right)} Z_{i, j}^{\left(2, m_{2}\right)} \\
\forall i, j=1, \ldots, s \text { and } \forall m_{1}, m_{2}=0,1, \ldots, M
\end{aligned}
$$

then it preserves quadratic invariants of (20) of the form $I\left(X^{(1)}, X^{(2)}\right)=X^{(1)^{\top}} D X^{(2)}$.

When the conditions (23) are fulfilled, the number of order conditions stated in Theorem 3 can be reduced. The key ingredient in the analysis is the Butcher product of two trees $u, v \in \tau_{1}, \tau_{2}, \ldots, \tau_{\kappa} \in$ $T \backslash\left\{\emptyset_{1}, \ldots, \emptyset_{Q}\right\}$. If

$$
u=\left[u_{1}, \ldots, u_{\kappa_{1}}\right]_{q_{1}, m_{1}}, \quad v=\left[v_{1}, \ldots, v_{\kappa_{2}}\right]_{q_{2}, m_{2}},
$$

the Butcher product is defined by

$$
u \circ v=\left[u_{1}, \ldots, u_{\kappa_{1}}, v\right]_{q_{1}, m_{1}},
$$

see Fig. 2. We can now state the following lemma:

Lemma 2. For Stratonovich SDEs of the form (20) we have for all $u \in T_{q_{1}, m_{1}}$ and $v \in T_{q_{2}, m_{2}}$

$$
\phi(u)(h) \cdot \phi(v)(h)=\phi(u \circ v)(h)+\phi(v \circ u)(h)
$$

for all $q_{1}, q_{2}=1,2$ and $m_{1}, m_{2}=0,1, \ldots, M$.

Proof. As for $u$ and $v$ given by (24) it holds

$$
\phi(u)(t)=\int_{0}^{t} \prod_{k_{1}=1}^{\kappa_{1}} \phi\left(u_{k_{1}}\right)(s) \circ \mathrm{d} W_{m_{1}}(s), \quad \phi(v)(t)=\int_{0}^{t} \prod_{k_{2}=1}^{\kappa_{2}} \phi\left(v_{k_{2}}\right)(s) \circ \mathrm{d} W_{m_{2}}(s),
$$

the product rule for Stratonovich integrals gives

$$
\begin{aligned}
\phi(u)(t) \cdot \phi(v)(t) & =\int_{0}^{t} \phi(v)(s) \prod_{k_{1}=1}^{\kappa_{1}} \phi\left(u_{k_{1}}\right)(s) \circ \mathrm{d} W_{m_{1}}(s) \\
& +\int_{0}^{t} \phi(u)(s) \prod_{k_{2}=1}^{\kappa_{2}} \phi\left(v_{k_{2}}\right)(s) \circ \mathrm{d} W_{m_{2}}(s) \\
& =\phi(u \circ v)(t)+\phi(v \circ u)(t) .
\end{aligned}
$$



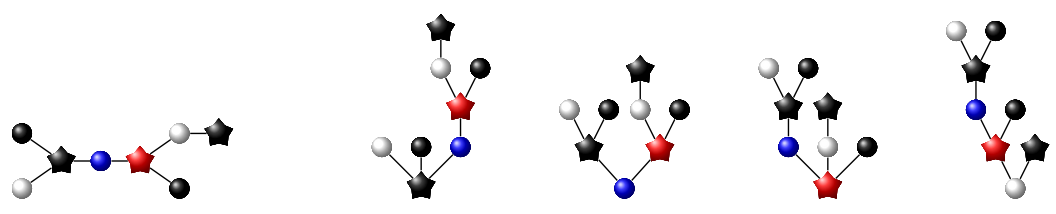

Figure 3. An unrooted tree $\hat{\tau}$ to the left, and some of the trees in $\widehat{T S}(\hat{\tau})$. The figure also illustrates the process described in the proof of Theorem 5 .

For $q_{1} \neq q_{2}$ this rule also holds for the weight functions of the numerical solution as stated in the following lemma:

Lemma 3. If an SPRK method of the form (3) satisfies the conditions in Theorem 4, then for all $u \in T_{1, m_{1}}$ and $v \in T_{2, m_{2}}$

$$
\Phi(u)(h) \cdot \Phi(v)(h)=\Phi(u \circ v)(h)+\Phi(v \circ u)(h)
$$

for all $m_{1}, m_{2}=0,1, \ldots, m$.

Proof. Multiply both sides of (23) in Theorem 4 by

$$
\prod_{k_{1}=1}^{\kappa_{1}} \Psi_{i}\left(u_{k_{1}}\right)(h) \prod_{k_{2}=1}^{\kappa_{2}} \Psi_{j}\left(v_{k_{2}}\right)(h)
$$

and sum over all $i, j=1, \ldots, s$,

$$
\begin{gathered}
\left(\sum_{i} \gamma_{i}^{\left(1, m_{1}\right)} \prod_{k_{1}=1}^{\kappa_{1}} \Psi_{i}\left(u_{k_{1}}\right)(h)\right)\left(\sum_{j} \gamma_{j}^{\left(2, m_{2}\right)} \prod_{k_{2}=1}^{\kappa_{2}} \Psi_{j}\left(v_{k_{2}}\right)(h)\right)= \\
\sum_{i, j}\left(\gamma_{j}^{\left(2, m_{2}\right)} Z_{j, i}^{\left(1, m_{1}\right)}+\gamma_{i}^{\left(1, m_{1}\right)} Z_{i, j}^{\left(2, m_{2}\right)}\right) \prod_{k_{1}=1}^{\kappa_{1}} \Psi_{i}\left(u_{k_{1}}\right)(h) \prod_{k_{2}=1}^{\kappa_{2}} \Psi_{j}\left(v_{k_{2}}\right)(h) .
\end{gathered}
$$

Using (12) and (13) completes the proof.

Let $T S$ be the set of trees for which a node of one shape will never be directly followed by a node of the same shape. For separable SDEs, all elementary differentials of trees in $T \backslash T S$ vanish. Given a $\tau \in T S$, let $\hat{\tau}$ be the corresponding unrooted tree, and let $\widehat{T S}(\hat{\tau}) \subset T S$ be the set of trees obtained from $\hat{\tau}$ by assigning one of the nodes as the root, see Fig. 3 for an illustration.

Theorem 5. Assume that (23) is satisfied. Let $\hat{\tau} \in \widehat{T S}$ be an unrooted tree of order $q \leq p$. If $\phi(\tau)(h)=\Phi(\tau)(h)+\mathcal{O}\left(h^{p+\frac{1}{2}}\right)$ for one rooted tree $\tau \in \widehat{T S}(\hat{\tau})$ and all rooted trees of order less than $q$, then it holds that $\phi(\tau)(h)=\Phi(\tau)(h)+\mathcal{O}\left(h^{p+\frac{1}{2}}\right)$ for all $\tau \in \widehat{T S}(\hat{\tau})$. Similarly, if $E \phi(\tau)(h)=$ $E \Phi(\tau)(h)+\mathcal{O}\left(h^{p+\frac{1}{2}}\right)$ for one rooted tree $\tau \in \widehat{T S}(\hat{\tau})$ and $\phi(\tau)(h)=\Phi(\tau)(h)+\mathcal{O}\left(h^{p+\frac{1}{2}}\right)$ for all rooted trees of order less than $q$, then it holds that $E \phi(\tau)(h)=E \Phi(\tau)(h)+\mathcal{O}\left(h^{p+\frac{1}{2}}\right)$ for all $\tau \in \widehat{T S}(\hat{\tau})$.

Proof. The argument is the same as for the non-partitioned case proven in $[3$. For trees with one node the theorem is trivially true. Let $\hat{\tau}$ be an unrooted tree of order $q$ and two or more nodes, and let $\tau$ be a corresponding rooted tree $\tau \in \widehat{T S}(\hat{\tau})$. Pick one branch $v$ from the root of $\tau$ and let the remaining part of $\tau$ be $u$, so that $\tau=u \circ v$. Then $u$ and $v$ have roots of different shapes, and Lemma 3 applies. Clearly, the orders of $u$ and $v$ are less than the order of $\tau$, and by Lemma 2 and the assumptions of the theorem we then have

$$
\phi(v \circ u)(h)=\Phi(v \circ u)(h)+\mathcal{O}\left(h^{p+\frac{1}{2}}\right)
$$


respectively

$$
E \phi(v \circ u)(h)=E \Phi(v \circ u)(h)+\mathcal{O}\left(h^{p+\frac{1}{2}}\right) .
$$

Because the choice of branch $v$ was arbitrary, this means that this condition is satisfied for all trees with the same graph as $\tau$, but with a root shifted to one of its neighboring nodes. A repeated use of this argument proves the result. The process is illustrated in Fig. 3 .

Example 4. A simple example for an SPRK method fulfilling (23) is the stochastic Störmer-Verlet method [14, given by the following tableau:

\begin{tabular}{cc|cc}
0 & 0 & 0 & 0 \\
$\frac{h}{2}$ & $\frac{h}{2}$ & $\frac{\Delta W_{m}}{2}$ & $\frac{\Delta W_{m}}{2}$ \\
\hline$\frac{h}{2}$ & 0 & $\frac{\Delta W_{m}}{2}$ & 0 \\
$\frac{h}{2}$ & 0 & $\frac{\Delta W_{m}}{2}$ & 0 \\
\hline \hline$\frac{h}{2}$ & $\frac{h}{2}$ & $\frac{\Delta W_{m}}{2}$ & $\frac{\Delta W_{m}}{2}$ \\
\hline$\frac{h}{2}$ & $\frac{h}{2}$ & $\frac{\Delta W_{m}}{2}$ & $\frac{\Delta W_{m}}{2}$
\end{tabular}.

To analyze its order, note that due to (20) being separable, the elementary differentials corresponding to trees for which a node of shape $q(q \in\{1,2\})$ is followed by a node of the same shape vanish. Taking into account Theorem 5, the remaining trees $\tau$ with $\rho(\tau) \leq \frac{3}{2}$ to be considered are then (for $q_{1}, q_{2} \in\{1,2\}$ with $\left.q_{1} \neq q_{2}\right)$

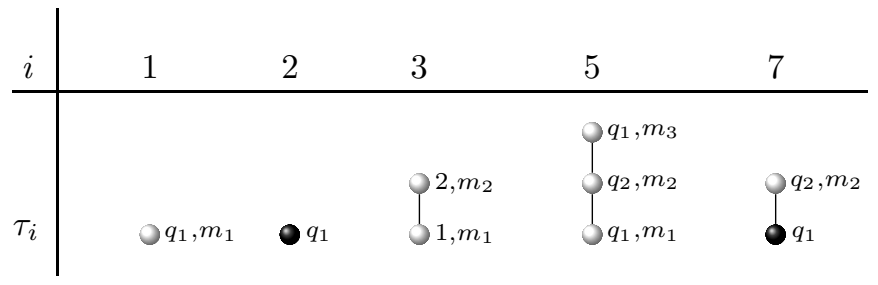

It is straightforward to show that $\phi(\tau)=\Phi(\tau)$ for trees $\tau_{1}, \tau_{2}$ in (25), and that this is true for $\tau_{3}$ only if $m_{1}=m_{2}$, while it holds also for $m_{1} \neq m_{2}$ that $E \phi\left(\tau_{3}\right)=E \Phi\left(\tau_{3}\right)$. Thus, for SDEs (20) with multidimensional noise, the above method is only of order $\frac{1}{2}$. For scalar noise, it holds also $\phi\left(\tau_{7}\right)=\Phi\left(\tau_{7}\right)$ and $E \phi\left(\tau_{5}\right)=E \Phi\left(\tau_{5}\right)$, but not $\phi\left(\tau_{5}\right)=\Phi\left(\tau_{5}\right)$, so in this case the method is of order 1. Similarly, it follows that the method is of weak order one for multidimensional noise.

The content of this section demonstrates the potential of the B-series formulation, in the sense that theory developed and well understood for ODEs here directly can be applied for SDEs. It also demonstrates how to use the particular structure of the problem at hand to reduce the set of order conditions that have to be satisfied. This is demonstrated in Table 2, But even if the number of conditions is reduced in this case, the conditions in Theorem 4 are still so restrictive that it is far from a trivial task to construct higher order methods.

\section{Conclusion}

In this paper we have developed a general B-series theory for SPRK methods. Such methods are rarely applied to general SDEs, they are more likely to be constructed for SDEs with certain structure. We have therefore emphasized how the general theory, summarized in Theorem 3 , can be modified to cover a few common examples of such cases. We hope other researchers can find the theory useful for constructing new methods, or to prove order results for applying existing methods to broader classes than originally constructed for. 
TABLE 2. Total number of trees up to order 2 for SDEs with scalar noise and two partitionings. Here, column (all) gives the number when assuming no restrictions, column (s.p.) the number counting only trees with nonzero elementary differentials for separable problems, and column (q.i.) the number of independent conditions if additionally the quadratic invariant condition is satisfied.

\begin{tabular}{crrr}
\hline$\rho$ & all & s.p. & q.i. \\
\hline 0.5 & 2 & 2 & 2 \\
1 & 6 & 4 & 3 \\
1.5 & 22 & 8 & 4 \\
2 & 92 & 20 & 9 \\
\hline Sum & 122 & 34 & 18 \\
\hline
\end{tabular}




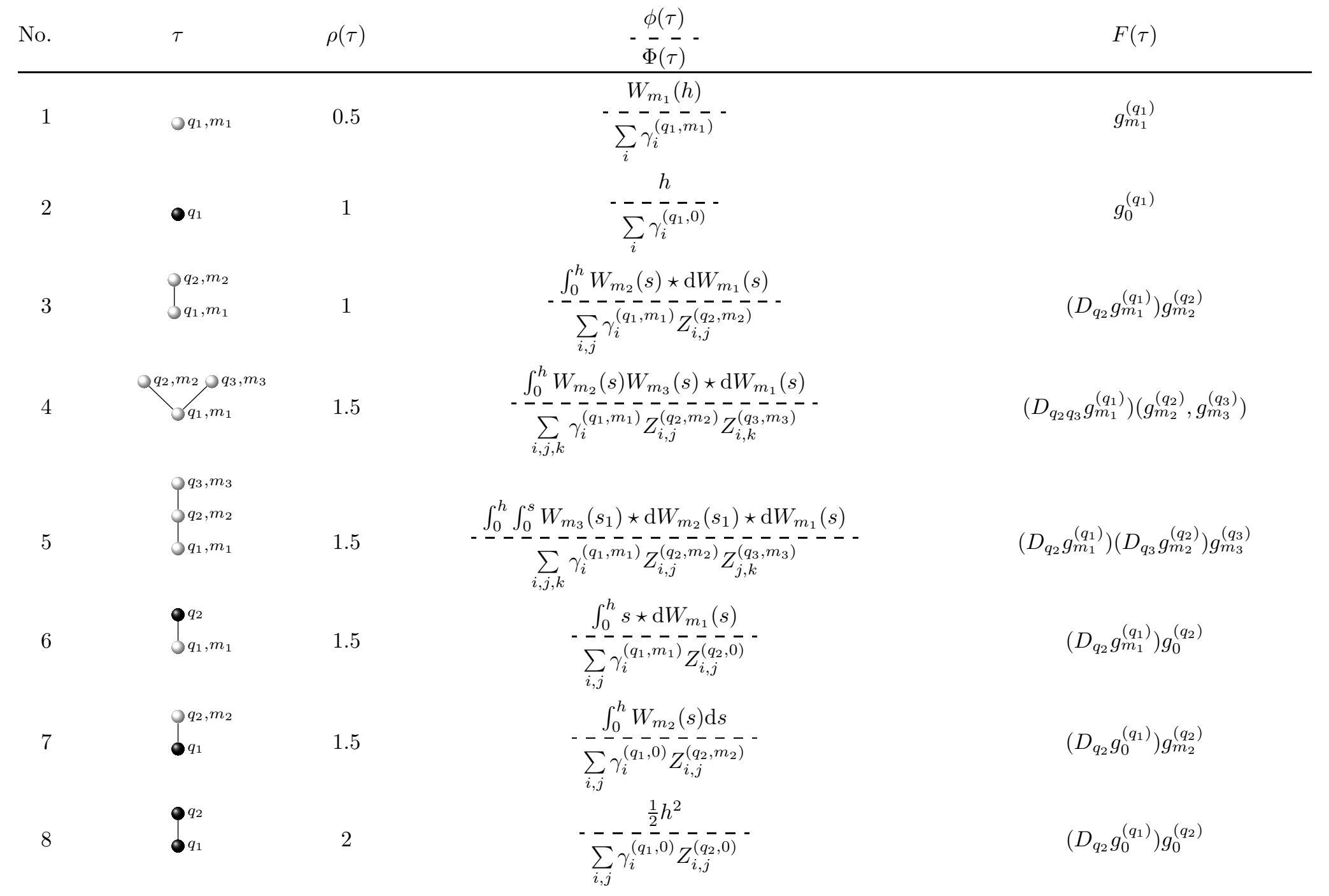


$q_{3}, m_{3}$

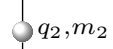

- $q_{1}$

$q_{3}, m_{3}$

12

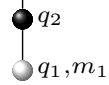

13

$$
q_{2}, m_{2}
$$

$q_{4}, m_{4}$

$q_{3}, m_{3}$

$q_{2}, m_{2}$

$q_{1}, m_{1}$

15

$q_{2}, m_{2} \rho q_{3}, m_{3}$

$$
-\frac{\int_{0}^{h} W_{m_{2}}(s) W_{m_{3}}(s) \mathrm{d} s}{\sum_{i, j, k} \gamma_{i}^{\left(q_{1}, 0\right)}-\overline{Z_{i, j}^{\left(q_{2}, m_{2}\right)}}-\overline{Z_{i, k}^{\left(q_{3}, m_{3}\right)}}-}
$$$$
\int_{0}^{h} W_{m_{2}}(s) s \star \mathrm{d} W_{m_{1}}(s)
$$$$
\sum_{i, j, k} \gamma_{i}^{\left(q_{1}, m_{1}\right)} Z_{i, j}^{\left(q_{2}, m_{2}\right)} Z_{i, k}^{\left(q_{3}, 0\right)}
$$

$$
-\frac{\int_{0}^{h} \int_{0}^{s} W_{m_{3}}\left(s_{1}\right) \star \mathrm{d} W_{m_{2}}\left(s_{1}\right) \star \mathrm{d} W_{m_{1}}(s)}{\sum_{i, j, k} \gamma_{i}^{\left(q_{1}, 0\right)} Z_{i, j}^{\left(q_{2}, m_{2}\right)} Z_{j, k}^{\left(q_{3}, m_{3}\right)}----}
$$

2

$$
-\frac{\int_{0}^{h} \int_{0}^{s}-W_{m_{3}}\left(s_{1}\right) \mathrm{d} s_{1} \star \mathrm{d} W_{m_{1}}(s)}{\sum_{i, j, k} \gamma_{i}^{\left(q_{1}, m_{1}\right)} \overline{Z_{i, j}^{\left(q_{2}, 0\right)}} \overline{Z_{j, k}^{\left(q_{3}, m_{3}\right)}}-}
$$

$$
-\frac{\int_{0}^{h} \int_{0}^{s} s_{1} \star \mathrm{d} W_{m_{2}}\left(s_{1}\right) \star \mathrm{d} W_{m_{1}}(s)}{\sum_{i, j, k} \gamma_{i}^{\left(q_{1}, m_{1}\right)} \overline{Z_{i, j}^{\left(q_{2}, m_{2}\right)}} \overline{Z_{j, k}^{\left(q_{3}, 0\right)}}}
$$

$\int_{0}^{h} \int_{0}^{s} \int_{0}^{s_{1}} \mathrm{~d} W_{m_{4}}\left(s_{2}\right) \star \mathrm{d} W_{m_{3}}\left(s_{2}\right) \star \mathrm{d} W_{m_{2}}\left(s_{1}\right) \star \mathrm{d} W_{m_{1}}(s)$

2 $\sum_{i, j, k, l} \gamma_{i}$

$\int_{0}^{h} W_{m_{2}}(s) \int_{0}^{s} W_{m_{4}}\left(s_{1}\right) \circ \mathrm{d} W_{m_{3}}\left(s_{1}\right) \circ \mathrm{d} W_{m_{1}}(s)$

$$
\left(D_{q_{2} q_{3}} g_{0}^{\left(q_{1}\right)}\right)\left(g_{m_{2}}^{\left(q_{2}\right)}, g_{m_{3}}^{\left(q_{3}\right)}\right)
$$

$$
\left(D_{q_{2} q_{3}} g_{m_{1}}^{\left(q_{1}\right)}\right)\left(g_{m_{2}}^{\left(q_{2}\right)}, g_{0}^{\left(q_{3}\right)}\right)
$$

$\left(D_{q_{2}} g_{0}^{\left(q_{1}\right)}\right)\left(D_{q_{2}} g_{m_{2}}^{\left(q_{2}\right)}\right) g_{m_{3}}^{\left(q_{3}\right)}$

$\left(D_{q_{2}} g_{m_{1}}^{\left(q_{1}\right)}\right)\left(D_{q_{3}} g_{0}^{\left(q_{2}\right)}\right) g_{m_{3}}^{\left(q_{3}\right)}$

$\left(D_{q_{2}} g_{m_{1}}^{\left(q_{1}\right)}\right)\left(D_{q_{3}} g_{m_{2}}^{\left(q_{2}\right)}\right) g_{0}^{\left(q_{3}\right)}$

$\left(D_{q_{2}} g_{m_{1}}^{\left(q_{1}\right)}\right)\left(D_{q_{3}} g_{m_{2}}^{\left(q_{2}\right)}\right)\left(D_{q_{4}} g_{m_{3}}^{\left(q_{3}\right)}\right) g_{m_{4}}^{\left(q_{4}\right)}$

$\left(D_{q_{2} q_{3}} g_{m_{1}}^{\left(q_{1}\right)}\right)\left(g_{m_{2}}^{\left(q_{2}\right)},\left(D_{q_{4}} g_{m_{3}}^{\left(q_{3}\right)}\right) g_{m_{4}}^{\left(q_{4}\right)}\right)$ 
16

$q_{4}, m_{4}, q_{3}, m_{3}, q_{2}, m_{2}$

$$
\int_{q_{1}, m_{1}}
$$

$q_{4}, m_{4} \rho q_{3}, m_{3}$

$$
q_{q_{1}, m_{1}}, m_{2}
$$

$\int_{0}^{h} W_{m_{4}}(s) W_{m_{3}}(s) W_{m_{2}}(s) \circ \mathrm{d} W_{m_{1}}(s)$ $\sum_{i, j, k, l} \gamma_{i}^{\left(\overline{q_{1}}, m_{1}\right)} \overline{Z_{i, j}^{\left(q_{2}, m_{2}\right)}} Z_{i, k}^{\left(q_{3}, m_{3}\right)} \overline{\left.Z_{i, l}^{\left(q_{4}\right.}, m_{4}\right)}-$

$\int_{0}^{h} \int_{0}^{s} W_{m_{4}}\left(s_{1}\right) W_{m_{3}}\left(s_{1}\right) \circ \mathrm{d} W_{m_{2}}\left(s_{1}\right) \circ \mathrm{d} W_{m_{1}}(s)$ $\sum_{i, j, k, l} \gamma_{i}^{\left(q_{1}, m_{1}\right)} Z_{i, j}^{\left(q_{q}, \bar{m}_{2}\right)} \overline{Z_{j, k}^{\left(q_{3}, m_{3}\right)}} Z_{j, l}^{\left(q_{4}, m_{4}\right)}-\cdots$
$\left(D_{q_{2} q_{3} q_{4}} g_{m_{1}}^{\left(q_{1}\right)}\right)\left(g_{m_{2}}^{\left(q_{2}\right)}, g_{m_{3}}^{\left(q_{3}\right)}, g_{m_{4}}^{\left(q_{4}\right)}\right)$

$\left(D_{q_{2}} g_{m_{1}}^{\left(q_{1}\right)}\right)\left(D_{q_{3} q_{4}} g_{m_{2}}^{\left(q_{2}\right)}\right)\left(g_{m_{3}}^{\left(q_{3}\right)}, g_{m_{4}}^{\left(q_{4}\right)}\right)$ 


\section{REFERENCES}

[1] L Abia and JM Sanz-Serna, Partitioned Runge-Kutta methods for separable Hamiltonian problems, Mathematics of computation 60 (1993), no. 202, 617-634.

[2] Markus Ableidinger, Evelyn Buckwar, and Harald Hinterleitner, A stochastic version of the Jansen and Rit neural mass model: Analysis and numerics, The Journal of Mathematical Neuroscience 7 (2017), no. 1, 8.

[3] Sverre Anmarkrud and Anne Kværn $\varnothing$, Order conditions for stochastic Runge-Kutta methods preserving quadratic invariants of Stratonovich SDEs, J. Comput. Appl. Math. 316 (2017), 40-46. MR 3588726

[4] Kevin Burrage and Pamela Marion Burrage, High strong order explicit Runge-Kutta methods for stochastic ordinary differential equations, Appl. Numer. Math. 22 (1996), no. 1-3, 81-101, Special issue celebrating the centenary of Runge-Kutta methods.

[5] - Order conditions of stochastic Runge-Kutta methods by B-series, SIAM J. Numer. Anal. 38 (2000), no. $5,1626-1646$.

[6] Kevin Burrage and Grant Lythe, Accurate stationary densities with partitioned numerical methods for stochastic differential equations, SIAM J. Numer. Anal. 47 (2009), no. 3, 1601-1618. MR 2505866 (2011a:60009)

[7] Pamela M. Burrage, Runge-Kutta methods for stochastic differential equations, Ph.D. thesis, The University of Queensland, Brisbane, 1999.

[8] John C. Butcher, Coefficients for the study of Runge-Kutta integration processes, J. Austral. Math. Soc. 3 (1963), 185-201. MR 0152129 (27 \#2109)

[9] Kristian Debrabant and Anne Kværn $\varnothing$, B-series analysis of stochastic Runge-Kutta methods that use an iterative scheme to compute their internal stage values, SIAM J. Numer. Anal. 47 (2008/09), no. 1, 181-203.

[10] Stochastic Taylor expansions: Weight functions of B-series expressed as multiple integrals, Stoch. Anal. Appl. 28 (2010), no. 2, 293-302.

[11] Niels Grønbech-Jensen and Oded Farago, A simple and effective Verlet-type algorithm for simulating Langevin dynamics, Molecular Physics 111 (2013), no. 8, 983-991.

[12] Ernst Hairer, Christian Lubich, and Gerhard Wanner, Geometric numerical integration, Springer Series in Computational Mathematics, vol. 31, Springer, Heidelberg, 2010.

[13] Darryl Holm and Tomasz Tyranowski, Stochastic discrete Hamiltonian variational integrators, (2016).

[14] Jialin Hong, Dongsheng $\mathrm{Xu}$, and Peng Wang, Preservation of quadratic invariants of stochastic differential equations via Runge-Kutta methods, Appl. Numer. Math. 87 (2015), 38-52.

[15] Peter E. Kloeden and Eckhard Platen, Numerical solution of stochastic differential equations, Applications of Mathematics (New York), vol. 23, Springer-Verlag, Berlin, 1992.

[16] Yoshio Komori, Multi-colored rooted tree analysis of the weak order conditions of a stochastic Runge-Kutta family, Appl. Numer. Math. 57 (2007), no. 2, 147-165.

[17] Yoshio Komori, Taketomo Mitsui, and Hiroshi Sugiura, Rooted tree analysis of the order conditions of ROW-type scheme for stochastic differential equations, BIT 37 (1997), no. 1, 43-66.

[18] Qiang Ma, Deqiong Ding, and Xiaohua Ding, Symplectic conditions and stochastic generating functions of stochastic Runge-Kutta methods for stochastic Hamiltonian systems with multiplicative noise, Appl. Math. Comput. 219 (2012), no. 2, 635-643.

[19] Qiang Ma and Xiaohua Ding, Stochastic symplectic partitioned Runge-Kutta methods for stochastic Hamiltonian systems with multiplicative noise, Applied Mathematics and Computation 252 (2015), 520 - 534.

[20] G. N. Milstein, Yu. M. Repin, and M. V. Tretyakov, Numerical methods for stochastic systems preserving symplectic structure, SIAM J. Numer. Anal. 40 (2002), no. 4, 1583-1604. MR 1951908

[21] G. N. Milstein and M. V. Tretyakov, Stochastic numerics for mathematical physics, Scientific Computation, Springer-Verlag, Berlin, 2004. MR 2069903

[22] Grigori N. Milstein, Numerical integration of stochastic differential equations, Mathematics and its Applications, vol. 313, Kluwer Academic Publishers Group, Dordrecht, 1995, Translated and revised from the 1988 Russian original. MR 1335454 (96e:65003)

[23] Grigori N. Milstein, Yu. M. Repin, and M. V. Tretyakov, Symplectic integration of Hamiltonian systems with additive noise, SIAM J. Numer. Anal. 39 (2002), no. 6, 2066-2088 (electronic).

[24] Bernt Øksendal, Stochastic differential equations, sixth ed., Universitext, Springer-Verlag, Berlin, 2003, An introduction with applications. MR 2001996 (2004e:60102)

[25] Andreas Rößler, Stochastic Taylor expansions for the expectation of functionals of diffusion processes, Stochastic Anal. Appl. 22 (2004), no. 6, 1553-1576.

[26] _ Rooted tree analysis for order conditions of stochastic Runge-Kutta methods for the weak approximation of stochastic differential equations, Stoch. Anal. Appl. 24 (2006), no. 1, 97-134.

[27] J. M. Sanz-Serna and L. Abia, Order conditions for canonical Runge-Kutta schemes, SIAM J. Numer. Anal. 28 (1991), no. 4, 1081-1096. 
[28] M. Seeßelberg, H. P. Breuer, H. Mais, F. Petruccione, and J. Honerkamp, Simulation of one-dimensional noisy Hamiltonian systems and their application to particle storage rings, Zeitschrift für Physik C Particles and Fields 62 (1994), no. 1, 63-73.

[29] Peng Wang, Jialin Hong, and Dongsheng Xu, Construction of symplectic Runge-Kutta methods for stochastic Hamiltonian systems, Commun. Comput. Phys. 21 (2017), no. 1, 237-270. MR 3579605

Faculty of Environmental Sciences and natural Resource Management, Norwegian University of Life SCIEnCES, 1433 Ås, Norway

Department of Mathematics and Computer Science, University of Southern Denmark, 5230 Odense M, DENMARK

Department of Mathematical Sciences, Norwegian University of Science and Technology, 7491 TrondHEIM, NORWAY 